\title{
Temperature and correlations in 1-dimensional systems
}

\author{
Claudio Giberti ${ }^{1, a}$, Lamberto Rondoni ${ }^{2, b}$, and Cecilia Vernia ${ }^{3, c}$ \\ 1 Dipartimento di Scienze e Metodi dell'Ingegneria, Università di Modena e Reggio E., Via Amendola \\ 2, Padiglione Morselli, I-42122 Reggio E., Italy \\ 2 Dipartimento di Matematica, Politecnico di Torino, corso Duca degli Abruzzi 24, I-10129, Torino, \\ Italy \\ 3 Dipartimento di Scienze Fisiche, Informatiche e Matematiche, Università di Modena e Reggio Emilia, \\ Via G. Campi 213/B, 41125 Modena, Italy
}

\begin{abstract}
Local thermodynamic equilibrium (LTE) plays a crucial role in statistical mechanics and thermodynamics. Under small driving and LTE, locally conserved quantities are transported as prescribed by linear hydrodynamic laws, in which the local material properties of the systems at hand are represented by the transport coefficients. The robustness and universality of equilibrium properties is not guaranteed in nonequilibrium states, in which different microscopic quantities may behave differently, even if they coincide at equilibrium. We investigate these issues considering 1-dimensional chains of $N$ oscillators. We observe that non-negligible fluctuations, and persistence of correlations frustrate the onset of LTE, hence the existence of thermodynamic fields, such as temperature.
\end{abstract}

\section{Introduction}

In their seminal paper Ref.[1], Rieder, Lebowitz and Lieb investigated the properties of 1dimensional systems made of $N$ harmonic oscillators, with only nearest neighbors and, at their ends, with stochastic heat baths. They proved that energy (interpreted as heat) flows from hot to cold baths and that the profile of kinetic energy (interpreted as temperature) decreases exponentially in the direction of the hotter bath, and in the bulk its slope vanishes in the $N \rightarrow \infty$ limit. Thus, globally energy flows from the hotter to the colder bath, while locally it goes from regions of lower kinetic energy to regions of higher kinetic energy, which is reminiscent of results on uphill diffusion, such as those obtained in Ref.[2] . Therefore, the kinetic energy and the energy flow do not behave in these harmonic 1-dimensional systems like temperature and heat would, in systems obeying thermodynamic laws.

With hindsight, this is not surprising, because thermodynamic behaviours belong to 3dimensional macroscopic objects, under the condition of Local Thermodynamic Equilibrium (LTE). Mechanical quantities, such as kinetic energy and energy flux cannot be identified with thermodynamic quantities, unless a number of conditions that make LTE possible, are

\footnotetext{
${ }^{a}$ e-mail: claudio.giberti@unimore.it

b e-mail: lamberto.rondoni@polito.it

c e-mail: cecilia.vernia@unimore.it
} 
verified, see for instance Refs. [3] §9, [4] Chapters 3, 4 and 5, [5] Section 15.1, [6] Section 2.3, [7] Section 3.3, [8] Chapter 1, [9, 10]. In particular, LTE holds if microscopic, mesoscopic and macroscopic space and time scales are well separated, so that object can be subdivided in mesoscopic cells that are very small compared to macroscopic sizes, but contain a very large number of particles. Consequently, boundary effects are negligible compared to bulk effects, and neighboring cells can practically be considered small isolated thermodynamic systems, which thermalize within very short times compared to the macroscopic scale. In other words, correlations decay very rapidly in space and time and fluctuations are negligible, from the macroscopic point of view. Fluctuations, in particular, are characteristic of particles systems: they are larger in size for larger systems, and they may be observed in macroscopic systems $[11,12]$, but they must be negligible on the scale of observation, for the thermodynamic laws to hold.

These may look almost contradictory conditions -how can mass and energy flow between isolated systems?- but they are actually realized in most phenomena occurring at the scales of our daily life, see $e . g$. [8] for an extensive illustration of these facts. For instance, the motion of the center of mass of a mesoscopic cell can be regular and minimally energetic compared to strongly irregular and exceedingly fast motion of the molecules inside the cell. The fact is that the amounts corresponding to macroscopic observations are minimal compared to those stored at the the microscopic levels. When this is the case, thermodynamic laws may apply, and conduction, associated with disordered motions, can be distinguished from convection, which is associated with regular motion, capable of doing mechanical work.

Provided the LTE condition is verified, matter behaves like a continuum, obeying thermodynamic laws, according to which the statistical laws are invariably and surely verified: in each single system heat flows without exceptions from hot to cold bodies; it is not the consequence of mere averaging procedures. Being robust under variations of macroscopic parameters such as boundary conditions, and requiring no knowledge of the microscopic conditions, such as the particles interaction potentials etc., thermodynamic laws are the rule at the space and time scales of our daily life.

At the nanometric scale, or in low dimensional systems (1D and 2D) the above is not guaranteed. For instance, transport coefficients in 1D systems strongly depend on the boundary conditions, instead of being local properties, as pertains to thermodynamic material properties [13-19]. Therefore, one commonly speaks of "anomalous" transport.

In this paper we investigate the issue of temperature, treated in many specialized works, cf. Refs.[20-26], focusing on chains of $N$ Lennard-Jones oscillators without on-site potentials, whose first and last oscillators interact with deterministic "heat" baths. To that purpose, we observe that energy equipartition holds in equilibrium homogeneous systems, so that the kinetic energy of any particle equals that of all other particles.

Consequence of equipartition is that numerous different microscopic notions of temperature are equivalent at equilibrium. For instance, the thermodynamic temperature is obtained averaging in time the average over all particles of the kinetic energy: temperature is indeed, a collective property belonging to a very large number of particles, measured on a macroscopic time scale, $[3,4,23,27,28]$. Nevertheless, in equilibrium the same result is obtained from the time average of the kinetic energy of a single particle, as well as from numerous other microscopic quantities, including configurational temperatures and combinations of configurational and kinetic temperatures $[20,29,30]$.

Away from equilibrium, the situation is more problematic. Equipartition is violated [26, 31,32], the statistic describing the state of the system is model dependent, and the ergodic properties of the particles dynamics are only partially understood [33,34]. Hence, there is no universally accepted microscopic notion of nonequilibrium temperature [20-26]. Analogous considerations hold for the definition of heat flux, that requires a clear distinction between energy transport due to macroscopic motions (convection), and transport without macroscopic motions (conduction), cf. Chapter 4 of Ref.[35], and Section III.2 and Chapter XI of Ref.[36]. 
To clarify these issues, we compare single particle with local mesoscopic quantities, and we distinguish two cases: a) the mesososcopic cells are fixed in space, as appropriate for solids; b) they move with the particles they contain, as in presence of convection, which seems to be our case, cf. Refs.[37,38]. We find that:

- two mesoscopic notions of kinetic temperature with fixed cells in space, $T^{C}$ and $K^{T}$, behave like the single particle kinetic temperature $T$, that depends on particle number and is not localized in space (see e.g. Ref.[1,15,39] for different single particle kinetic temperature profiles);

- one, in principle more appropriate, mesoscopic notion of kinetic temperature, based on the peculiar velocities within moving cells, $T^{p}$, does not agree with $T, T^{C}$ and $K^{T}$, although the time average of the velocity of every particle in the cell, hence the cell itself, vanishes;

- cell and molecular motion are correlated, making convection hard to be distinguished from conduction, hence kinetic energy hard to be linked to temperature. Indeed, the motion of a single particle influences its neighbors, producing a kind of convective cascade.

- the validity of the local virial relation does not imply the existence of the temperature field.

That difficulties do not ease -they actually seem to worsen [40] - when $N$ grows at fixed boundary temperatures, indicates that LTE, hence thermodynamic quantities such as temperature, can hardly be established under our conditions in 1D systems such as ours. A possible consequence is that the standard hydrodynamic limit may not apply to systems like the one discussed here, even in presence of stochastic heat baths. Indeed, the existence of the hydrodynamic limit implies the validity of LTE [6]. This, however, does not exclude the existence of the hydrodynamic limit for different 1D systems or under different boundary conditions.

\section{Chains of oscillators and "temperature" profiles}

Consider a 1D chain of $N$ identical moving particles of equal mass $m$, and positions $x_{i}$, $i=1, \ldots, N$. Add two particles with fixed positions, $x_{0}=0$ and $x_{N+1}=(N+1) a$, where $a>0$ is the lattice spacing. Let nearest neighbors interact via the Lennard-Jones potential $(\mathrm{LJ})$ :

$$
V_{1}(r)=\epsilon\left[\left(\frac{a}{r}\right)^{12}-2\left(\frac{a}{r}\right)^{6}\right],
$$

where $r$ is the distance between nearest neighbors: $r=x_{i}-x_{i-1}$ and $\epsilon>0$ is the depth of the potential well. Thus, $x_{i}=a i$, with $i=0, \ldots, N+1$, is a configuration of stable mechanical equilibrium for the system. We also consider interactions involving first and second nearest neighbors, with second potential given by [41]:

$$
V_{2}(s)=\epsilon\left[\left(\frac{2 a}{s}\right)^{12}-2\left(\frac{2 a}{s}\right)^{6}\right]
$$

where $s=x_{i}-x_{i-2}$. Further, we add two particles with fixed positions $x_{-1}=-a$ and $x_{N+2}=(N+2) a$. With potential $V=V_{1}+V_{2}$, the system has the usual stable mechanical equilibrium configuration $x_{i}=a i, i=-1, \ldots, N+2$. The first and last moving particles are in contact with two Nosé-Hoover thermostats, at kinetic temperatures $T_{L}$ (on the left) and $T_{R}$ (on the right) and with relaxation times $\theta_{L}$ and $\theta_{R}$. Introducing the forces

$$
F_{1}(r)=\frac{\partial V_{1}}{\partial r}(r), \quad F_{2}(s)=\frac{\partial V_{2}}{\partial s}(s)
$$


the equations of motion are given by:

$$
\begin{aligned}
& m \ddot{x}_{1}=F_{1}\left(x_{1}\right)-F_{1}\left(x_{2}-x_{1}\right)-\xi_{1} \dot{x}_{1}, \\
& m \ddot{x}_{i}=F_{1}\left(x_{i}-x_{i-1}\right)-F_{1}\left(x_{i+1}-x_{i}\right), \quad i=2, \ldots, N-1, \\
& m \ddot{x}_{N}=F_{1}\left(x_{N}-x_{N-1}\right)-F_{1}\left(x_{N+1}-x_{N}\right)-\xi_{N} \dot{x}_{N},
\end{aligned}
$$

with

$$
\dot{\xi}_{1}=\frac{1}{\theta_{L}^{2}}\left(\frac{m \dot{x}_{1}^{2}}{T_{L}}-1\right), \quad \dot{\xi}_{N}=\frac{1}{\theta_{R}^{2}}\left(\frac{m \dot{x}_{N}^{2}}{T_{R}}-1\right),
$$

in the case of nearest neighbors interaction. For first and second neighbors interactions, we have:

$$
\begin{aligned}
& m \ddot{x}_{1}=F_{1}\left(x_{1}\right)-F_{1}\left(x_{2}-x_{1}\right)+F_{2}\left(x_{1}+a\right)-F_{2}\left(x_{3}-x_{1}\right)-\xi_{1} \dot{x}_{1}, \\
& m \ddot{x}_{2}=F_{1}\left(x_{2}-x_{1}\right)-F_{1}\left(x_{3}-x_{2}\right)+F_{2}\left(x_{2}\right)-F_{2}\left(x_{4}-x_{2}\right)-\xi_{2} \dot{x}_{2}, \\
& m \ddot{x}_{i}=F_{1}\left(x_{i}-x_{i-1}\right)-F_{1}\left(x_{i+1}-x_{i}\right)+F_{2}\left(x_{i}-x_{i-2}\right)-F_{2}\left(x_{i+2}-x_{i}\right), \quad i=3, \ldots, N-2, \\
& m \ddot{x}_{N-1}=F_{1}\left(x_{N-1}-x_{N-2}\right)-F_{1}\left(x_{N}-x_{N-1}\right)+F_{2}\left(x_{N-1}-x_{N-3}\right)-F_{2}\left(x_{N+1}-x_{N-1}\right)-\xi_{N-1} \dot{x}_{N-1}, \\
& m \ddot{x}_{N}=F_{1}\left(x_{N}-x_{N-1}\right)-F_{1}\left(x_{N+1}-x_{N}\right)+F_{2}\left(x_{N}-x_{N-2}\right)-F_{2}\left(x_{N+2}-x_{N}\right)-\xi_{N} \dot{x}_{N},
\end{aligned}
$$

with

$$
\begin{aligned}
& \dot{\xi}_{l}=\frac{1}{\theta_{L}^{2}}\left(\frac{m \dot{x}_{l}^{2}}{T_{L}}-1\right), l=1,2, \\
& \dot{\xi}_{l}=\frac{1}{\theta_{R}^{2}}\left(\frac{m \dot{x}_{l}^{2}}{T_{R}}-1\right), l=N-1, N .
\end{aligned}
$$

The hard-core nature of the LJ potentials preserves the order of particles: $0<x_{1}<x_{2}<$ $\cdots<x_{N}<(N+1) a$ holds at all times, if it does at the initial time [42].

For such systems, a form of single particle virial relation is found to hold [43] (see also the upper panel of Fig. 6), and then the average kinetic energy of a single particle is often taken as the temperature $T_{i}$ in the position occupied by particle $x_{i}$ [45]:

$$
T_{i}=\left\langle\frac{p_{i}{ }^{2}}{m}\right\rangle, \quad i=1, \ldots, N .
$$

Here, $p_{i}$ is the momentum of particle $i$, the angular brackets $\langle\cdot\rangle$ denote time average, and $T_{i}$ is called single particle kinetic temperature. In the case in which $T_{L} \neq T_{R}$, the single particle kinetic temperature profile may take rather peculiar forms, compared to the linear thermodynamic temperature profiles in homogeneous solids when Fourier law holds. This is illustrated in great detail in the specialized literature, cf. [15,39,45-48] just to cite a few. Certain profiles define kinds of universality classes, that can be analytically expressed in the infinite $N$ limit [39]. At the same time, numerically simulated profiles of various kinds of 1D systems, appear to be sensitive to all parameters, such as the relaxation constants of the thermostats, the interaction parameters etc. [15]. This is not surprising, since many correlations persist in space and time in low dimensional systems, making the various quantities non-local, thus hindering the realization of LTE [14,49-52].

In the following sections, we report our results about systems with various numbers of particles $N$. The parameters defining the Lennard-Jones potentials are $\epsilon=1$ and $a=1$, while the mass of the particles is $m=1$. The relaxation times of the thermostats $\theta_{L}$ and $\theta_{R}$ are set to 1 . The numerical integrator used is the fourth-order Runge-Kutta method with step size $10^{-3}$. The time averages are typically taken over $O\left(10^{8}\right)-O\left(10^{9}\right)$ time steps in the stationary state. 


\section{Comparing with mesoscopic notions of temperature}

At equilibrium, the thermodynamic temperature is given by the time average of the instantaneous average over all particles of the kinetic energy. In such average, by definition, particles with high kinetic energy contribute to high temperature, and regions with a larger number of particles give a greater contribution than regions of equal volume with a smaller number of particles. However, equilibrium is a homogeneous state in which all particles and equal volumes equally contribute to the thermodynamic properties $[53,54]$. In LTE cases, this picture is replicated on the scale of mesoscopic cells that are large compared to particles interaction ranges, and small compared to the observation scale [3,8-10,27]. Then, the average over all particles is replaced by averages over the particles that lie in such (practically isolated) cells. In presence of temperature gradients, equipartition is violated and different particles or different regions must contribute differently to the local temperatures. Moreover, particles may enter and exit a given cell, contributing at times to a cell, and at other times to other cells. Under LTE, such boundary effects are negligible by definition [3,8], but in general, especially in $1 \mathrm{D}$ driven systems, they must be dealt with.

Unfortunately, there is no universally valid strategy for that, apart from the principle that temperature must be higher in one region than in an another, if energy flows from the first to the latter. What this means in dynamical terms is far from obvious, when the microscopic statistics are not standard, something that happens, for instance, in presence of dissipation. Various possibilities may thus be considered [20,23,24,30].

The most direct mesoscopic extension of the global equilibrium kinetic temperature is the cell kinetic temperature defined as follows: subdivide the interval $[0,(N+1) a]$ in cells $C_{k}=[(N+1) a(k-1) / M,(N+1) a k / M], k=1, \ldots, M$, that are sufficiently large to house a large number of particles. The borders of these cells are fictitious: particles can move freely back and forth through them. At a given instant of time $\tau$, let the number of particles in cell $C_{k}$ be denoted by $n_{k}(\tau)$ and introduce the quantity:

$$
\widehat{T}_{k}(\tau)=\frac{1}{n_{k}(\tau)} \sum_{i=1}^{N} \frac{1}{2} m \dot{x}_{i}^{2}(\tau) \chi_{k}\left(x_{i}(\tau)\right),
$$

where $\chi_{k}\left(x_{i}(\tau)\right)$ equals 1 if the particle $i$ position at time $\tau, x_{i}(\tau)$, belongs to $C_{k}$, and it vanishes if $x_{i}(\tau) \notin C_{k}$. The quantity $\widehat{T}_{k}(\tau)$ is the instantaneous mean kinetic energy per particle in $C_{k}$, at time $\tau$. Averaging $\widehat{T}_{k}(\tau)$ in time, we obtain the cell kinetic temperature, i.e. time average of the instantaneous mean kinetic energy per particle in $C_{k}$ :

$$
\begin{aligned}
T^{C}(k) & =\frac{1}{\tau_{\max }} \sum_{\tau=1}^{\tau_{\max }} \widehat{T}_{k}(\tau) \\
& =\frac{1}{\tau_{\max }} \sum_{\tau=1}^{\tau_{\max }} \frac{1}{n_{k}(\tau)} \sum_{i=1}^{N} \frac{1}{2} m \dot{x}_{i}^{2}(\tau) \chi_{k}\left(x_{i}(\tau)\right)
\end{aligned}
$$

where $\tau_{\max }$ is the total number of time steps [55]. Because of equipartition, at equilibrium $T^{C}$ constitutes a valid local counterpart of the global instantaneous mean kinetic energy per particle, which in turn equals the single particle kinetic temperature $T_{i}$. The quantity $T^{C}(k)$ is, however, quite different from $T_{i}$, because:

a) it is mesoscopic, as required by thermodynamics;

b) it depends on space rather than on particle label.

Nevertheless, for chains with first and second neighbors Lennard-Jones interaction potential, we find that the $T^{C}$ profiles are approximately equal to the $T_{i}$ profiles. Figure 1 shows that increasing the number of cells, $M$, when $N$ is sufficiently large, makes $T^{C}$ better and better 

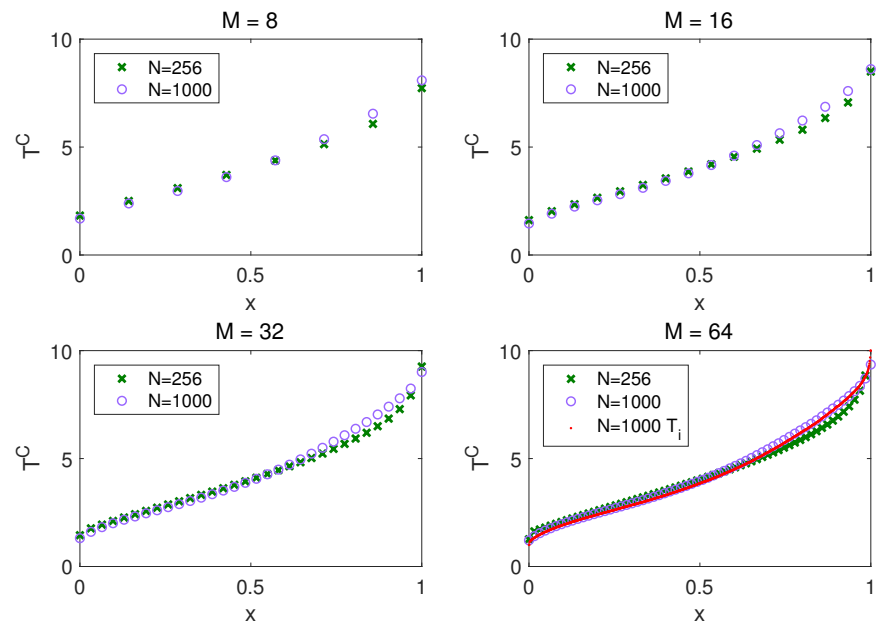

Fig. 1. $T^{C}(k)$ profile as a function of the rescaled cell position $x=k / M$, for $N=256$ (green crosses) and $N=1000$ (purple circles), with $M=8,16,32,64, T_{L}=1$ and $T_{R}=10$. Although $T^{C}$ is a function of space, not of particle label, it converges to the single particle kinetic temperature profile, see the data for $N=1000$ in the panel for $M=64$, where $T_{i}$ (red dots) is represented vs $x=i / N$.

reproduce the $T_{i}$ profile. This happens despite the fact the particle positions undergo $O(N)$ fluctuations, as discussed in Ref.[40].

Therefore, the convergence of $T^{C}$ to $T_{i}$, that can be appreciated also in the upper panel of Fig.2, must be related to the fact that nearby particles collectively and coherently oscillate like one block [49], with similar kinetic energies. In that case, it does not matter which of them is in $C_{k}$; the average over the particles inside $C_{k}$ approximately equals the single particle kinetic energy. This, however, is not what happens in the case of thermodynamic equilibrium, in which the equality of the particle energies is a statistical effect concerning particles that move incoherently and in a very disordered fashion. For chains with third nearest neighbor interactions, we reached the same conclusions, which indicates that they do not depend on any special choice of interaction potentials.

Because of the normalization to the total time in Eq.(12), and not to the time spent inside $C_{k}$, the contribution of one particle with given energy to $T_{C}(k)$ is small if the particle spends a short time in $C_{k}$, and it is larger in cells in which it stays longer. At the same time, this notion of "temperature" does not care whether many or just a few particles visit the cell of interest, since only the average energy over the particles visiting $C_{k}$ matters. Thus, the energy contributed by the particles to cell $C_{k}$ is not additive and, in the event that all particles visiting $C_{k}$ spend a short time in it, the time averaging of (12) yields a low cell temperature $T_{C}(k)$; this is not impossible in non-homogeneous states. However, in principle, even a short residence time may suffice for a considerable amount of energy to flow out of $C_{k}$, thanks to suitable interactions or if a large number of particle escapes from that cell. From the point of view of Fourier law, the temperature of the cell should then be larger rather than smaller of that in the neighboring cells, but $T_{C}(k)$ does not guarantee this result.

As in 1D systems such as ours one cannot ensure the good statistics needed for LTE [4,53, 54], the probability of such extreme events may not be negligible. To take into account these situations, it is necessary to enhance the contribution of a particle that spends a limited time in cell $C_{k}$, conditioning temporal averages to such a time. This has been done in Refs.[56, 57], for systems of non-interacting particles, such as billiards. To treat systems of interacting particles, we modify the temperature of Refs. [56,57] as follows. Take a long observation time interval and let $u(i, k)$ be the cardinality of $I(i, k)$, the set of time steps in which particle $i$ 
lies in cell $C_{k}$. Compute first the time average of the kinetic energy of particle $i$, conditioned to the time that this particle has spent in cell $C_{k}$ :

$$
K(i, k)=\frac{1}{u(i, k)} \sum_{\tau \in I(i, k)} \frac{1}{2} m \dot{x}_{i}^{2}(\tau),
$$

then average over the particles that have visited cell $C_{k}$, using as a weight the time spent by each of them in $C_{k}$ :

$$
\begin{aligned}
K^{T}(k) & =\frac{1}{\sum_{i=1}^{N} u(i, k)} \sum_{i=1}^{N} u(i, k) K(i, k) \\
& =\frac{1}{\sum_{i=1}^{N} u(i, k)} \sum_{i=1}^{N} \sum_{\tau \in I(i, k)} \frac{1}{2} m \dot{x}_{i}^{2}(\tau) .
\end{aligned}
$$

The quantity $K^{T}(k)$ is a weighted average, over the particles that have visited $C_{k}$, of their conditional time averaged kinetic energy $K(i, k)$. The time average of the kinetic energy of particle $i$ is obtained dividing by the time that $i$ spends in the cell, not the total time, hence the contribution of particle $i$ to $K^{T}(k)$ may be large even if the time it spent in $C_{k}$ is short. This suffices to conclude that $K^{T}(k)$ differs from both $T^{C}(k)$ and $T_{i}$.

What about the distinction between conduction and convection? To discuss this issue, one should first define the collective motion of a cell, and the motion of particles in it, with respect to the cell motion. This can be done, for instance, subdividing the chain of $N$ oscillators in $M$ cells made of consecutive particles, letting cell $\widehat{\mathcal{C}_{\ell}}$ contain the particles $\frac{N}{M}(\ell-1)+1 \leq$ $i \leq \frac{N}{M} \ell, \ell=1, \ldots, M$, when $n_{c}=\frac{N}{M}$ is an integer. One may then introduce the velocity of the center of mass of $\widehat{\mathcal{C}}_{\ell}, v_{G_{\ell}}$ say, as the velocity of the cell, and the peculiar velocity of the particles in $\widehat{\mathcal{C}_{\ell}}$ :

$$
c_{i}=\dot{x}_{i}-v_{G_{\ell}} \quad \text { if } \quad i \in \widehat{\mathcal{C}_{\ell}} .
$$

In accord with this definition we introduce the peculiar temperature of cell $\ell$ as:

$$
T_{\ell}^{p}=\frac{1}{\tau_{\max }} \sum_{\tau=1}^{\tau_{\max }} \frac{1}{2 n_{c}} \sum_{i \in \widehat{\mathcal{C}}_{\ell}} m c_{i}(\tau)^{2}
$$

which reduces to the cell kinetic temperature, if the centers of mass of the cells are at rest.

In a case with $N=2000, T_{L}=1$ and $T_{R}=10$, we have computed the following time averages: $\left\langle v_{G_{\ell}}\right\rangle,\left\langle v_{G_{\ell}}^{2}\right\rangle,\left\langle c_{i}\right\rangle,\left\langle c_{i}^{2}\right\rangle,\left\langle v_{i} v_{G_{\ell}}\right\rangle$ and $\left\langle T_{i}\right\rangle$. We found that both $\left\langle v_{G_{\ell}}\right\rangle$ and $\left\langle c_{i}\right\rangle$ vanish, consistently with the fact that all particles are bounded by still walls at $x_{0}=0$ and $x_{N+1}=$ $(N+1) a$. In Fig.2, upper panel, we compare the single particle kinetic temperature $T_{i}$, the cell kinetic temperature $T^{C}$ and the peculiar temperature $T^{p}$. In the lower panel, we then show the relative difference of the two mesoscopic quantities $T^{C}$ and $T^{p}$, i.e. $\left|T^{C}-T^{p}\right| / T^{p}$, which shows that they are similar but not identical. This result is further confirmed by Fig.3, showing that the relative differences range between $2 \%$ and $8 \%$.

In Fig.4, we note that the profiles of the square velocities $\left\langle v_{G_{\ell}}^{2}\right\rangle$ and $\left\langle c_{i}^{2}\right\rangle$ are similar but on different scales, and that the proportionality factor between the two approximately equals the mass inside a moving cell. Therefore, the kinetic energy of a cell, seen as a fluid element of mass equal to the sum of the masses of its particles and that moves at the speed of its center of mass, is comparable to, instead of much smaller than, the total kinetic energy of the particles in the reference frame of this center of mass. This observation is related to the following: the normalized correlations between the velocity of a particle in a cell and the 

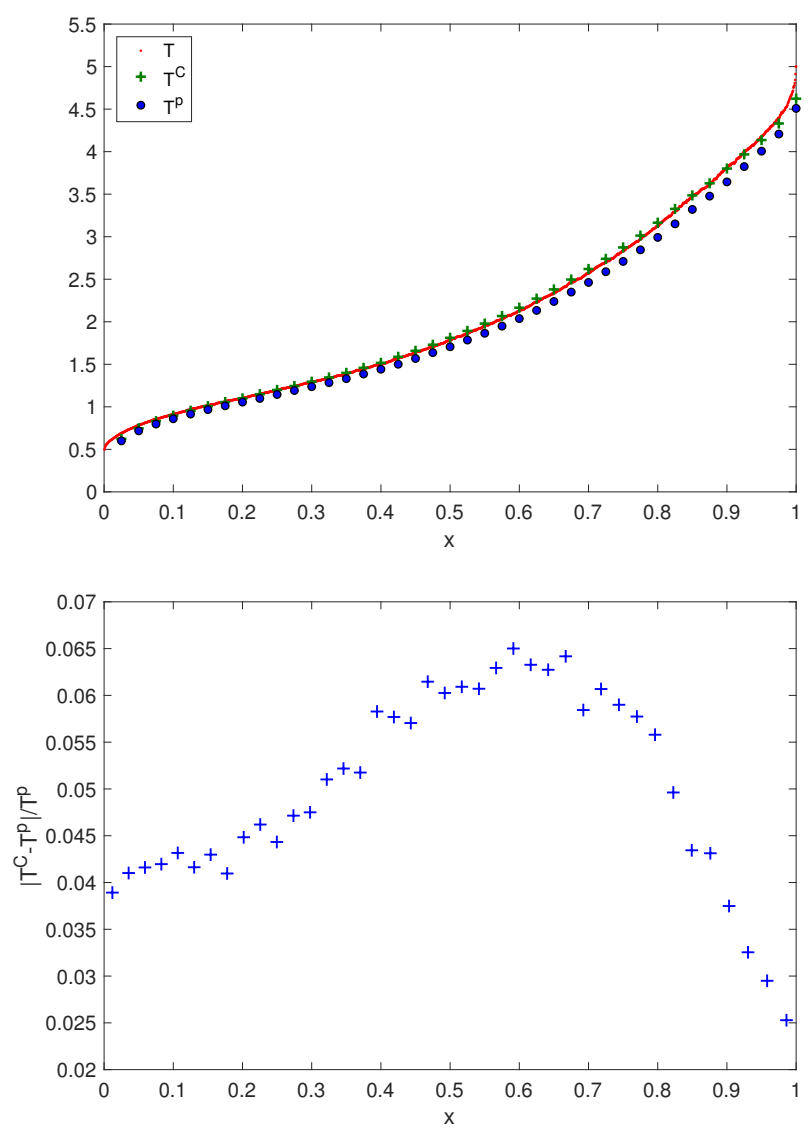

Fig. 2. $N=2000, T_{L}=1, T_{R}=10$. Upper panel: single particle kinetic temperature $T_{i}$ (red dots) vs $x=i / N$, cell temperature $T^{C}(k)$ (green crosses) and peculiar temperature $T^{p}(k)$ (blue bullets) vs $x=k / M$, with $M=40$. Lower panel: relative difference of the two mesoscopic quantities in the upper panel $\left|T^{C}-T^{p}\right| / T^{p}$. This difference ranges between $2.5 \%$ and $6.5 \%$.

velocity of the cell itself,

$$
\frac{\left\langle v_{i} v_{G_{\ell}}\right\rangle}{\sqrt{\left\langle v_{i}^{2}\right\rangle\left\langle v_{G_{\ell}}^{2}\right\rangle}}
$$

are rather large in general, and of about 15\% in the case of Fig.4, as illustrated by the upper panel of Fig.5. Moreover, the profile of the correlations has the same shape of $\left\langle c_{i}^{2}\right\rangle$ or $\left\langle v_{G_{\ell}}^{2}\right\rangle$ (compare with Fig.4). This fact is quantified by the lower panel of Fig.5, in which $\left\langle v_{i} v_{G_{\ell}}\right\rangle$ is given as a function of $\left\langle c_{i}^{2}\right\rangle$, and fitted to the line

$$
\left\langle v_{i} v_{G_{\ell}}\right\rangle \simeq a\left\langle c_{i}^{2}\right\rangle+b
$$

Note that, for macroscopic systems in which LTE holds, the notions of temperature considered here are equivalent: energy equipartition implies that all degrees of freedom contribute the same, so that all averages eventually lead to the thermodynamic temperature. In our 1D systems, the correlations that persist in time, and our observations concerning Figs. 4,2 and 5 imply that this is not the case. 

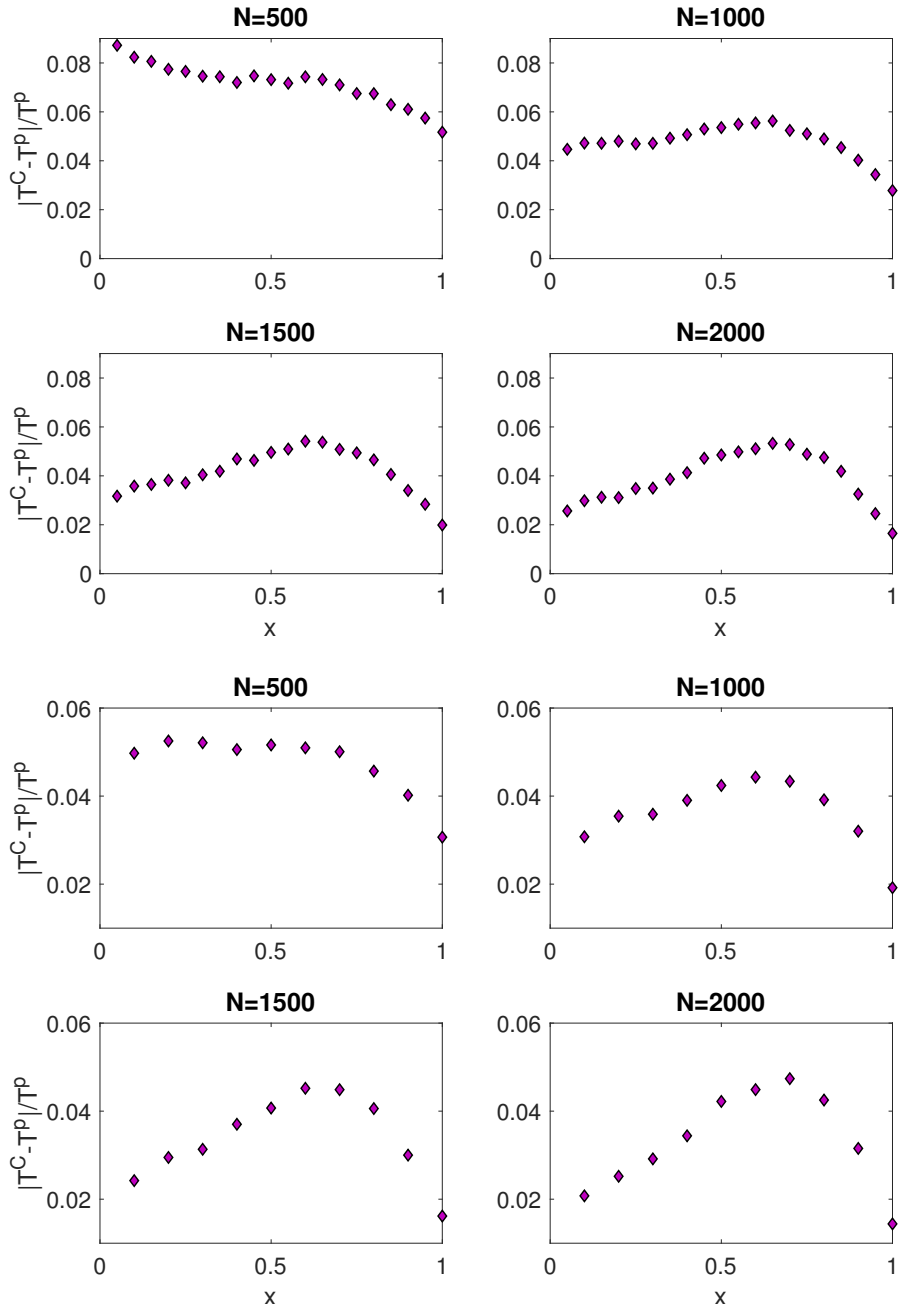

Fig. 3. Further examples of relative deviations between cell temperature and peculiar temperature $\left|T^{C}-T^{p}\right| / T^{p}$ computed for $N=500,1000,1500,2000$. In the uppermost four panels the number of cells $M$ is 20 , while in the lower panels it is 10 .

\section{Concluding remarks}

In this work we have presented numerical results concerning several kinds of 1D systems of nonlinear oscillators, in contact with two Nosé-Hoover thermostats. Scrutinizing the behaviour of mechanical quantities that are commonly considered in the specialized literature, we have investigated the violations of LTE, and the fragility (non-universality) of "thermodynamic" quantities, that is expected in 1D systems [15, 14,23].

Thermodynamic properties emerge from the collective behavior of very large assemblies of interacting particles, if correlations decay rapidly compared to observation time scales, and if boundary effects are negligible. While this is often the case of 3D mesoscopic cells containing large numbers of properly interacting particles, it is not obvious in 1D systems.

Considering the mesoscopic "temperatures" $T^{C}$ and $K^{T}$, we have shown that grouping particles together in cells localized in space does not suffice to obtain profiles that differ from the single particle kinetic temperature, that is not a function of space, but of particle label. 

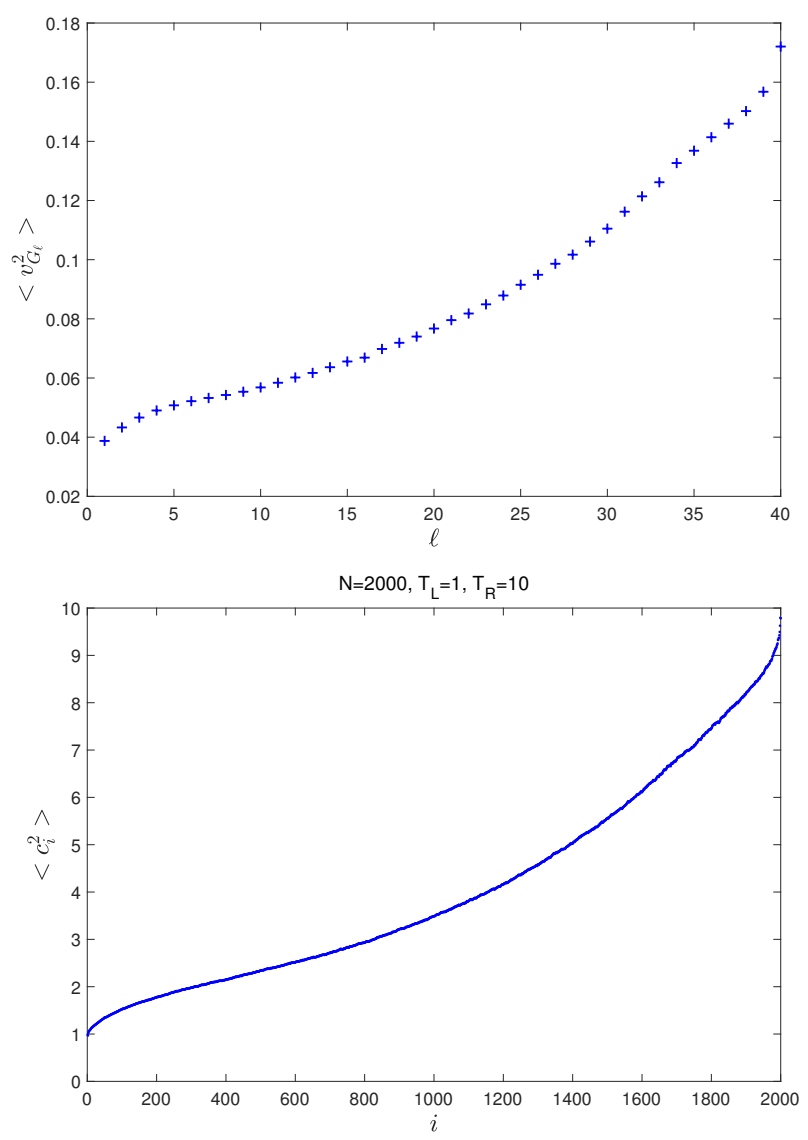

Fig. 4. Simulations for $N=2000, T_{L}=1, T_{R}=10$ with $M=40$ cells, hence with 50 particles in each cell. Upper panel: average squared velocity of center of mass of $\left\langle v_{G_{\ell}}^{2}\right\rangle$ in cells $\ell=1, \ldots, M$ versus $\ell$. Lower panel: average squared peculiar velocity $\left\langle c_{i}^{2}\right\rangle$ for particles $i=1, \ldots, N$ versus $i$. The two quantities are not sufficiently separated. The quantities $v_{G_{\ell}}^{2}$ and $c_{i}^{2}$ are not sufficiently separated, compared to thermodynamic cases.

On the other hand, the profiles of the mesosocpic "temperature" $T^{p}$ do substantially differ from those of the previously mentioned temperatures. Therefore, one concludes that results obtained using one or the other must be taken with a grain of salt.

The fact is that large fluctuations [40] and persisting correlations prevent LTE and the direct identification of mechanical quantities with thermodynamic ones. As a matter of fact, even the validity of the local virial relation, see the upper panel of Fig. 6, does not imply the statistics of square velocities corresponding to a temperature, cf. Fig.6, lower panel. Therefore, the standard laws and quantities of macroscopic systems are not appropriate to understand the behavior of 1D systems such as our chains of Lennard-Jones oscillators. Future developments of our work will focus on large $N$ limits that reproduce mesoscopic rather than macroscopic length scales as, for instance, in [58-60]. 

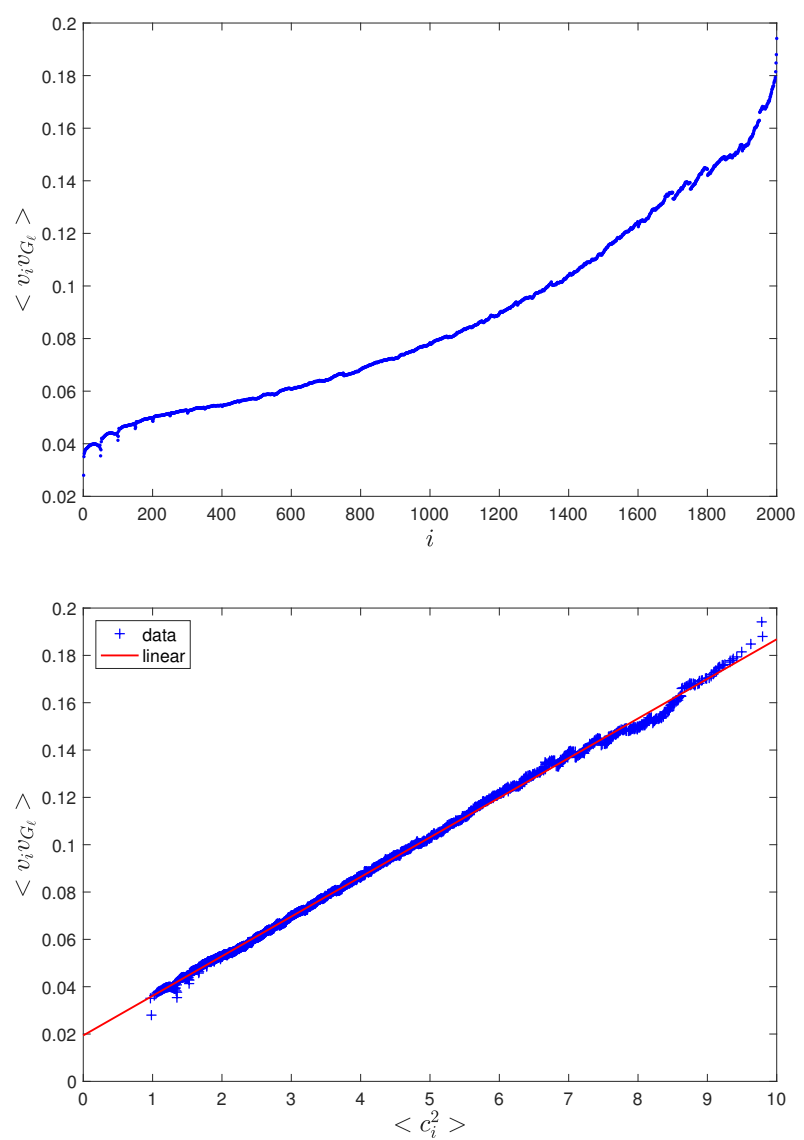

Fig. 5. $N=2000, T_{L}=1, T_{R}=10$ with $M=40$ cells. Upper panel: the correlations $\left\langle v_{i} v_{G_{\ell}}\right\rangle$ for $i=1, \ldots N$ between the velocity of particle $i$ and the center of mass of the cell containing $i$. Lower panel: $\left\langle v_{i} v_{G_{\ell}}\right\rangle$ vs $\left\langle c_{i}^{2}\right\rangle$ and a linear fit.

\section{Acknowledgments}

The authors are grateful to Carlos Mejia-Monasterio for enlightening remarks. This work is partially supported by Gruppo Nazionale per la Fisica Matematica (GNFM-INdAM). C.G. and C.V. acknowledge financial supports from "Fondo di Ateneo per la Ricerca 2015" and "Fondo di Ateneo per la Ricerca 2016"- Università di Modena e Reggio Emilia. LR acknowledges partial support from MIUR grant Dipartimenti di Eccellenza 2018-2022.

\section{References}

1. Z. Rieder Z, J.L. Lebowitz, E. Lieb, Properties of a harmonic crystal in a stationary nonequilibrium state, J. Math. Phys. 81073 (1967)

2. M. Colangeli, C. Giardinà , C. Giberti, C. Vernia, Nonequilibrium two-dimensional Ising model with stationary uphill diffusion, Phys. Rev. E 97, 030103(R) (2018)

3. L.D. Landau, E.M. Lifshitz, Statistical Physics Volume 5 of Course of Theoretical Physics, Part 1, Pergamon Press, Oxford (1980)

4. S. Chibbaro, L. Rondoni, A. Vulpiani, Reductionism, Emergence and Levels of Reality, Springer Verlag, New York (2014) 

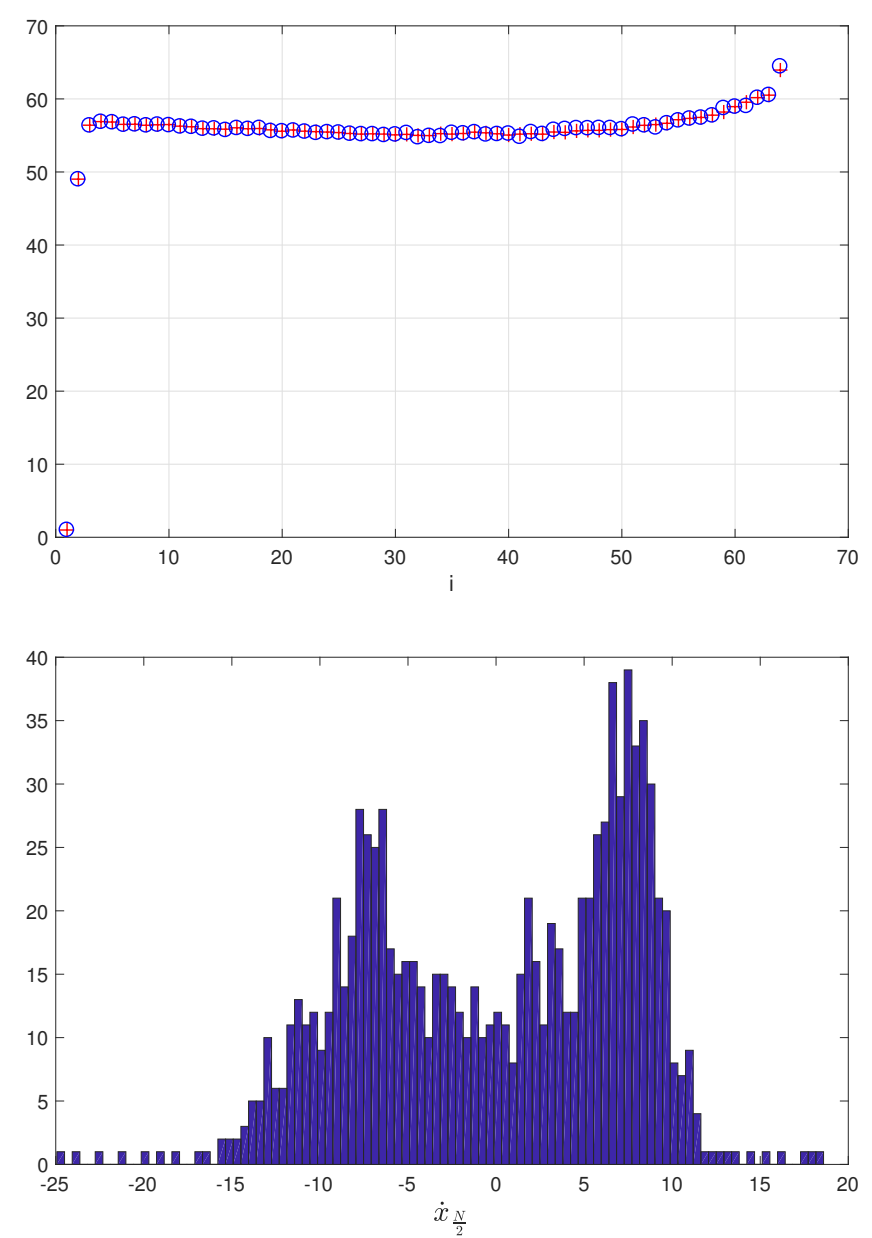

Fig. 6. Simulations for $N=64$, with nearest neighbors interaction, $T_{L}=1, T_{R}=64$. Upper panel: Average kinetic temperature $T_{i}$ (red crosses) and the local virial $\left\langle f_{i} x_{i}\right\rangle$, where $f_{i}$ is the total force acting on particle $x_{i}$ (blue circles). Note that the local virial property $\left\langle p_{i}^{2} / m\right\rangle=\left\langle f_{i} x_{i}\right\rangle$ is satisfied. Lower panel: histogram of $\dot{x}_{N / 2}$. This figure reveals a non maxwellian distribution for the velocity, showing the violation of LTE.

5. D. Kondepudi, I. Prigogine, Modern thermodynamics : from heat engines to dissipative structures, John Wiley \& Sons Ltd, Chichester (1998)

6. H. Spohn, Large Scale Dynamics of Interacting Particles, Texts and Monographs in Physics, Springer-Verlag, Heidelberg (1991)

7. J. Bellissard, Coherent and Dissipative Transport in Aperiodic Solids: An Overview, in P. Garbaczewski R. Olkiewicz (Eds.), Dynamics of Dissipation, Springer Verlag, Berlin (2002)

8. H.J. Kreuzer, Nonequilibrium thermodynamics and its statistical foundations, Claredon Press, Oxford (1981)

9. M. Falcioni, L. Palatella, S. Pigolotti, L. Rondoni, A. Vulpiani, Initial growth of Boltzmann entropy and chaos in a large assembly of weakly interacting systems, Physica A 385170 (2007)

10. L. Rondoni, S. Pigolotti, On $\Gamma-$ and $\mu$-space descriptions: Gibbs and Boltzmann entropies of symplectic coupled maps, Phys. Scr. 86058513 (2012)

11. M. Bonaldi et al., Nonequilibrium Steady-State Fluctuations in Actively Cooled Resonators, Phys. Rev. Lett. 103, 010601 (2009) 
12. L. Conti, M. Bonaldi, L. Rondoni, RareNoise: non-equilibrium effects in detectors of gravitational waves Class. Quantum Gravity, 27084032 (2010)

13. C. W. Chang, D. Okawa, H. Garcia, A. Majumdar, A. Zettl, Breakdown of Fouriers Law in Nanotube Thermal Conductors, Phys. Rev. Lett. 101075903 (2008); X. Xu et al., Length-dependent thermal conductivity in suspended single-layer graphene, Nature Comm. 5:3689, 1 (2014)

14. O.G. Jepps and L. Rondoni, Thermodynamics and complexity of simple transport phenomena J. Phys. A 39, 1311 (2006)

15. C. Giberti and L. Rondoni, Anomalies and absence of local equilibrium, and universality, in onedimensional particles systems, Phys. Rev. E 83, 041115 (2011)

16. S. Chen, Y. Zhang, J. Wang, H. Zhao, Key role of asymmetric interactions in low- dimensional heat transport, J. Stat. Mech. 033205 (2016)

17. S. Chen, Y. Zhang, J. Wang, H. Zhao, Diffusion of heat, energy, momentum, and mass in onedimensional systems, Phys. Rev. E 87, 032153 (2013)

18. M. Onorato, L. Vozella, D. Proment, Y.V. Lvov, Route to thermalization in the $\alpha$-Fermi-Pasta-Ulam system, Proc. Natl. Acad. Sci. U S A 112(14) 42084213 (2015)

19. A. Dhar and K. Saito, Heat conduction in the disordered Fermi-Pasta-Ulam chain, Phys. Rev. E 78 061136 (2008)

20. G.P. Morriss, L. Rondoni, Definition of temperature in equilibrium and nonequilibrium systems, Phys. Rev. E 59 R5 (1999)

21. J. Casas-Vzquez, D. Jou, Temperature in non-equilibrium states: a review of open problems and current proposals, Rep. Prog. Phys. 661937 (2003)

22. D. Jou, L. Restuccia, Caloric and entropic temperatures in non-equilibrium steady states, Physica A 460246 (2016)

23. D.J. Jou, J. Casas-Vàzquez, G. Lebon, Extended Irreversible Thermodynamics, Springer, New York (2010)

24. M. Criado-Sancho, D. Jou, J. Casas-Vzquez, Nonequilibrium kinetic temperatures in flowing gases, Phys. Lett. A 350339 (2006)

25. X. Cao and D. He, Interfacial thermal conduction and negative temperature jump in onedimensional lattices, Phys. Rev. E 92032135 (2015)

26. A. Puglisi, A. Sarracino, A. Vulpiani, 2017 Temperature in and out of equilibrium: A review of concepts, tools and attempts Phys. Rep. 709-710, 1 (2017)

27. K. Huang, Statistical Mechanics, John Wiley \& Sons, New York (1963)

28. G. Gallavotti, Statistical Mechanics, Springer, New York (1999)

29. H.H. Rugh, A dynamical approach to temperature, Phys. Rev. Lett. 78772 (1997)

30. O.G. Jepps, L. Rondoni, Deterministic thermostats, theories of nonequilibrium systems and parallels with the ergodic condition, J. Phys. A 43133001 (2010)

31. L. Conti et al, Effects of breaking vibrational energy equipartition on measurements of temperature in macroscopic oscillators subject to heat flux, J. Stat. Mech. P12003 (2013)

32. J.M. Ortiz de Zrate, J.V. Sengers, Hydrodynamic Fluctuations in Fluids and Fluid Mixtures, Elsevier, Amsterdam (2006)

33. G. Gallavotti, Nonequilibrium and Irreversibility, Springer, New York (2014)

34. D.J. Evans, S.R. Williams, D.J. Searles, L. Rondoni, On Typicality in Nonequilibrium Steady States, J. Stat. Phys. 164842 (2016)

35. M.W. Zemansky and R.H. Dittman, Heat and Thermodynamics, McGraw-Hill, New York (1997)

36. S.R. de Groot and P. Mazur, Non-equilibrium Thermodynamics, Dover, New York (1984)

37. O. Narayan and S. Ramaswamy, Anomalous heat conduction in one-dimensional momentumconserving systems, Phys. Rev. Lett. bf 89200601 (2002)

38. T. Mai and O. Narayan, Universality of one-dimensional heat conductivity, Phys. Rev. E 73061202 (2006)

39. L. Delfini, S. Lepri, R. Livi, A. Politi, Phys. Rev. E 73, 060201(R) (2006). S. Lepri, C. MejíaMonasterio, A. Politi, J. Phys. A 43, 065002 (2010); L. Delfini, S. Lepri, R. Livi, C. MejíaMonasterio, A. Politi, ibid. 43, 145001 (2010).

40. C. Giberti, L. Rondoni, C. Vernia, O(N)-fluctuations and lattice distortions in 1-dimensional systems, arXiv:1706.02886 [cond-mat.stat-mech] (2018)

41. P. De Gregorio, L. Rondoni, M. Bonaldi and L. Conti, Phys. Rev. B 84, 224103 (2011). L. Conti, P. De Gregorio, M. Bonaldi, A. Borrielli, M. Crivellari, G. Karapetyan, C. Poli, E. Serra, R.K. Thakur and L. Rondoni Phys. Rev. E 85, 066605 (2012) 
42. In some cases, we extended the Lennard-Jones interaction to the third nearest neighbors, preserving the equilibrium configuration $x_{i}=i a$. The corresponding equations of motion and thermostats are the natural modification of the previous ones, hence are not reported here.

43. In Ref.[44] a nonequilibrium mesoscopic version of the virial relation in given.

44. G. Falasco, F. Baldovin, K. Kroy, M. Baiesi, Mesoscopic virial equation for nonequilibrium statistical mechanics, New J. Phys. 18093043 (2016)

45. S. Lepri, R. Livi and A. Politi, Thermal conduction in classical low-dimensional lattices, Phys. Rep. 377, 1-80 (2003)

46. S. Lepri, R. Livi and A. Politi, Phys. Rev. Lett., 78 (10), 1896-1899 (1997); S. Lepri, R. Livi and A. Politi, Phsica D 119, 140-147 (1998)

47. A. Dhar, Heat Transport in low-dimensional systems, Advances in Physics, Vol. 57, No. 5, 457-537 (2008)

48. S.Lepri (Ed.), Thermal transport in low dimensions - From Statistical Physics to Nanoscale Heat Transfer, Lecture Notes in Physics, vol. 921, Springer, Heidelberg (2016)

49. P.I. Hurtado, Breakdown of hydrodynamics in a simple one-dimensional fluid, Phys. Rev. Lett. 96 010601 (2006)

50. S.J. Davie, O. G. Jepps, L. Rondoni, J. C. Reid, D. J. Searles, Phys. Script. 89, 048002 (2014)

51. O. Jepps, C. Bianca, L. Rondoni CHAOS 18, 013127 (2008). C. Bianca and L. Rondoni CHAOS 19, 013121 (2009)

52. L. Salari, L. Rondoni, C. Giberti, R. Klages, A simple non-chaotic map generating subdiffusive, diffusive and superdiffusive dynamics, CHAOS 25, 073113 (2015)

53. A.I. Khinchin, Mathematical Foundations of Statistical Mechanics, Dover Publications, New York (1949)

54. S. Chibbaro, L. Rondoni, A. Vulpiani, On the Foundations of Statistical Mechanics: Ergodicity, Many Degrees of Freedom and Inference, Commun. Theor. Phys. 62469 (2014)

55. In continuous time, the sum is merely replaced by an integral. We keep the sum, because we refer to numerical simulations.

56. D. Alonso, R. Artuso, G. Casati, I. Guarneri, Heat Conductivity and Dynamical Instability, Phys. Rev. Lett. 82 (9) 1859-1862 (1999)

57. B. Li, L. Wang and B. Hu, Finite Thermal Conductivity in 1D Models Having Zero Lyapunov Exponents, Phys. Rev. Lett. 88 (22) 223901 (2002)

58. M. Colangeli, L. Rondoni, Fluctuations in quantum one-dimensional thermostatted systems with off-diagonal disorder, J. Stat. Mech. Theor. Exp. P02009 (2013).

59. M. Colangeli, M. Pizzi, L. Rondoni, Current in a quantum driven thermostatted system with offdiagonal disorder, Physica A 3922977 (2013).

60. T. Vanzan, L. Rondoni, Quantum thermostatted disordered systems and sensitivity under compression, Physica A 493370 (2018). 